\title{
Resonance Contributions to $\eta$ Photoproduction on Protons Found Using Dispersion Relations and an Isobar Model
}

\author{
I.G.Aznauryan \\ Yerevan Physics Institute, Alikhanian Brothers St.2, Yerevan, 375036 Armenia \\ (e-mail addresses: aznaury@jlab.org, aznaur@jerewan1.yerphi.am)
}

\begin{abstract}
The contributions of the resonances $D_{13}(1520), S_{11}(1535), S_{11}(1650), D_{15}(1675), F_{15}(1680)$, $D_{13}(1700), P_{11}(1710), P_{13}(1720)$ to $\gamma p \rightarrow \eta p$ are found from the data on cross sections, beam and target asymmetries using two approaches: fixed-t dispersion relations and an isobar model. Utilization of the two approaches and comparison of the results obtained with different parametrizations of the resonance contributions allowed us to make conclusions about the model-dependence of these contributions. We conclude that the results for the contributions of the resonances $D_{13}(1520), S_{11}(1535), F_{15}(1680)$ to corresponding multipole amplitudes are stable. With this the results for $D_{13}(1520)$ and $F_{15}(1680)$, combined with their PDG photoexcitation helicity amplitudes, allowed us to find the branching ratios $\operatorname{Br}\left(D_{13}(1520) \rightarrow \eta N\right)=0.05 \pm 0.02 \%, B r\left(F_{15}(1680) \rightarrow \eta N\right)=0.16 \pm 0.04 \%$ which have significantly better accuracy than the PDG data. The total Breit-Wigner width of the $S_{11}(1535)$ is model-dependent, we have obtained $\Gamma\left(S_{11}(1520)\right)=142 \mathrm{MeV}$ and $195 \mathrm{MeV}$ using dispersion relations and the isobar model, respectively. The results for the $S_{11}(1650), D_{15}(1675), P_{11}(1710), P_{13}(1720)$ are model dependent, only the signs and orders of magnitude of their contributions to multipole amplitudes are determined. The results for the $D_{13}(1700)$ are strongly model-dependent.
\end{abstract}

PACS numbers: 13.60.Le, 14.20.Gk, 11.55.Fv, 11.80.Et, 25.20.Lj, 25.30.Rw 


\section{Introduction}

It is well known that photo- and electroproduction of $\eta$ on nucleons provide a unique opportunity for detailed study of the properties of the $S_{11}(1535)$ resonance, because this resonance has a large branching ratio into the $\eta N$ channel, unlike other resonances with close masses: $D_{13}(1520), S_{11}(1650), D_{15}(1675), F_{15}(1680)$, which overlap with the $S_{11}(1535)$ in $\pi$ photoproduction. The situation with overlapping resonances is significantly simplified in the case of $\eta$ in comparison with $\pi$ also for the reason that the resonances with isotopic spin $3 / 2$ do not contribute to $\eta$ photoproduction.

Investigation of $\eta$ photoproduction is interesting also for the following reasons. Investigating the contributions of the resonances with small $\eta N$ branching ratios and well known photocouplings, we have the possibility of specifying these branching ratios. Eta photoproduction provides also the possibility of searching for the "missing resonances", which cannot be observed in $\pi N$ scattering and $\pi$ photoproduction on nucleons.

The approaches which are used for extraction of resonance properties from experimental data can be divided into two groups. One group is based on coupled channels calculations, which has been used mostly to analyse $\pi N$ data $[1,2,3]$. The other group consists of the approaches which extract resonance properties from single reaction data by modeling background and parametrizing the resonance contributions to multipole amplitudes according to the Breit-Wigner formula. For the $\pi N$ scattering such an analysis is made in Ref. [4]. For the pion photo- and electroproduction on the nucleons the latest analyses of this type are done in Refs. [5, 6].

In the case of $\eta$ photoproduction on protons, there is an analysis made within the coupled channel approach based on effective Lagrangians [7]. At energies above $1.54 \mathrm{GeV}$ only sparse data on the differential cross sections were used in this analysis. More recent data on the differential cross sections from GRAAL [8] and CLAS [9], and the polarized beam asymmetry from GRAAL [10] were not available at that time. A more complete data set $[8,10,11,12]$ was analysed in Refs. $[13,14]$ using isobar models with parametrization of the resonance contributions according to the Breit-Wigner formula. 
The background in Ref. [13] is built from $s$ and $u$ channel nucleon contributions (the Born term) and $t$ channel vector meson exchanges. The model of Ref. [14] is based directly on the utilization of the quark model results. $t$ channel vector meson exchanges are excluded in this model in order to avoid possible double counting of contributions from the $s$ and $t$ channels; the background is built from the $u$ channel resonance contributions.

In this paper we will investigate the data on $\eta$ photoproduction on protons from Refs. $[8,9,10,11,12]$ using fixed- $t$ dispersion relations for invariant amplitudes. The imaginary parts of the amplitudes we will build, as in isobar models, from the $s$ channel resonance contributions parametrized in the Breit-Wigner form. Using dispersion relations we will find the real parts of the amplitudes, which include the contributions of the nucleon poles in the $s$ and $u$ channels (the Born term). They include also the integrals over the imaginary parts of the resonance contributions. As a result, in addition to the $s$ channel contributions, $u$ channel resonance contributions to the real parts of the amplitudes will be reproduced due to the crossing symmetry. Also, the point connected with the $t$ channel vector meson contributions does not arise in the dispersion relations approach. They do not directly enter the dispersion relations. Such contributions could be imitated by high energy contributions to the dispersion integrals. However, our estimations made for pion photoproduction on nucleons, where we have enough information for the estimation of these contributions, show that their role in the description of the data in the second and third resonance regions is negligibly small [15]. The dispersion relations approach will be presented in Sec. 2. In this paper we will present also our results on the description of the same data set in an isobar model which is very close to the model of Ref. [13]. The difference lies only in the slightly different parametrization of the resonance contributions and in the inclusion in our analysis of the Roper resonance $P_{11}(1440)$. This approach will be presented in Sec. 3. Comparison of the results obtained in the two approaches using the same data set will allow us to make conclusions on the model-dependence of the extracted resonance characteristics. We will compare also with the results obtained in Ref. [13] using an isobar model. This will allow us to check the dependence on the details of the model, and on the observables used in the analysis. The results obtained 
will be presented and discussed in Sec. 4. The conclusions are presented in Sec. 5 .

\section{Dispersion relations}

In the dispersion relations approach we use fixed-t dispersion relations for invariant amplitudes. All formulas in this paper we will write for the $\eta$ electroproduction on the nucleons, i.e. for the reaction $\gamma^{*} N \rightarrow \eta N$. The amplitudes of this reaction we choose following the work [16] in accordance with the definition of the hadron electromagnetic current in the form:

$$
\begin{array}{r}
I^{\mu}=\bar{u}\left(p_{2}\right) \gamma_{5}\left\{\frac{B_{1}}{2}\left[\gamma^{\mu}(\gamma \tilde{k})-(\gamma \tilde{k}) \gamma^{\mu}\right]+2 P^{\mu} B_{2}+2 \tilde{q}^{\mu} B_{3}+2 \tilde{k}^{\mu} B_{4}\right. \\
\left.-\gamma^{\mu} B_{5}+(\gamma \tilde{k}) P^{\mu} B_{6}+(\gamma \tilde{k}) \tilde{k}^{\mu} B_{7}+(\gamma \tilde{k}) \tilde{q}^{\mu} B_{8}\right\} u\left(p_{1}\right),
\end{array}
$$

where $\tilde{k}, \tilde{q}, p_{1}, p_{2}$ are 4-momenta of the virtual photon, $\eta$, initial and final nucleons, respectively, $P=\frac{1}{2}\left(p_{1}+p_{2}\right), B_{1}, B_{2}, \ldots B_{8}$ are invariant amplitudes which are functions of the invariant variables $s=\left(\tilde{k}+p_{1}\right)^{2}, t=(\tilde{k}-\tilde{q})^{2}, Q^{2} \equiv-\tilde{k}^{2}$.

The conservation of the hadron electromagnetic current leads to the relations:

$$
\begin{aligned}
4 Q^{2} B_{4} & =(s-u) B_{2}-2\left(t+Q^{2}-m_{\eta}^{2}\right) B_{3}, \\
2 Q^{2} B_{7} & =-2 B_{5}^{\prime}-\left(t+Q^{2}-m_{\eta}^{2}\right) B_{8},
\end{aligned}
$$

where $B_{5}^{\prime} \equiv B_{5}-\frac{1}{4}(s-u) B_{6}$. So, only the six of the eight invariant amplitudes are independent. As independent amplitudes let us choose $B_{1}, B_{2}, B_{3}, B_{5}^{\prime}, B_{6}, B_{8}$. For all these amplitudes unsubtracted dispersion relations at fixed $t$ can be written. The relations between $B_{1}, B_{2}, B_{3}, B_{5}^{\prime}, B_{6}, B_{8}$ and the multipole amplitudes and observables are given in Appendix A.

Unlike the $\pi$ electroproduction, in the case of $\eta$, dispersion relations for $p$ and $n$ are independent from each other and can be written separately. By this reason we write dispersion relations for $N$, supposing that $N=p$ or $n$ :

$$
\operatorname{Re} B_{i}^{N}\left(s, t, Q^{2}\right)=\quad e g_{\eta N N} R_{i}^{N}\left(Q^{2}\right)\left(\frac{1}{s-m_{N}^{2}}+\frac{\eta_{i}}{u-m_{N}^{2}}\right)
$$




$$
+\frac{P}{\pi} \int_{s_{c u t}}^{\infty} \operatorname{Im} B_{i}^{N}\left(s^{\prime}, t, Q^{2}\right)\left(\frac{1}{s^{\prime}-s}+\frac{\eta_{i}}{s^{\prime}-u}\right) d s^{\prime},
$$

where $R_{i}^{N}\left(Q^{2}\right)$ correspond to the residues in the nucleon poles:

$$
\begin{aligned}
& R_{1}^{N}\left(Q^{2}\right)=F_{1}^{N}\left(Q^{2}\right)+2 m_{N} F_{2}^{N}\left(Q^{2}\right) \\
& R_{2}^{N}\left(Q^{2}\right)=-F_{1}^{N}\left(Q^{2}\right) \\
& R_{3}^{N}\left(Q^{2}\right)=-\frac{1}{2} F_{1}^{N}\left(Q^{2}\right) \\
& R_{5}^{N^{\prime}}\left(Q^{2}\right)=\frac{m_{\eta}^{2}-Q^{2}-t}{2} F_{2}^{N}\left(Q^{2}\right) \\
& R_{6}^{N}\left(Q^{2}\right)=2 F_{2}^{N}\left(Q^{2}\right) \\
& R_{8}^{N}\left(Q^{2}\right)=F_{2}^{N}\left(Q^{2}\right)
\end{aligned}
$$

$e^{2} / 4 \pi=1 / 137, g_{\eta N N}$ is the $\eta N N$ pseudoscalar coupling constant, and $F_{1}^{N}\left(Q^{2}\right), F_{2}^{N}\left(Q^{2}\right)$ are the nucleon Pauli form factors. In the case of the real photons these form factors are normalized to $F_{1}^{p}(0)=1, F_{1}^{n}(0)=0, F_{2}^{N}(0)=\frac{\kappa_{N}}{2 m_{N}}, \kappa_{p}=1.79, \kappa_{n}=-1.91$.

The factors $\eta_{i}$ in the dispersion relations define the crossing symmetry properties of the invariant amplitudes, they are equal to $\eta_{1}=\eta_{2}=\eta_{6}=1, \eta_{3}=\eta_{5}^{\prime}=\eta_{8}=-1$.

Other distinctive feature of the dispersion relations for $\eta$ electroproduction is the presence in the dispersion integrals of the unphysical region from $s=s_{\text {cut }}=\left(m_{N}+m_{\pi^{0}}\right)^{2}$ to $s=s_{t h r}=\left(m_{N}+m_{\eta}\right)^{2}$. In this region we take into account the contribution of the Roper resonance $P_{11}(1440)$. Also, we continue to this region the contributions of $S_{11}(1535)$ and $S_{11}(1650)$ in the way which will be described below. In the unphisical region of the dispersion integrals, we do not take into account contributions of other resonances with higher masses as they are strongly suppressed in comparison with the contribution of $S_{11}(1535)$.

The contribution of the Roper resonance $P_{11}(1440)$ to dispersion integrals was found by evaluating the Feynman diagrams for $\gamma^{*}+N \rightarrow R \rightarrow \eta+N$ and the corresponding invariant amplitudes using the effective Lagrangians:

$$
\mathcal{L}_{\gamma N R}^{(1)}=-e F_{1}^{R}\left(Q^{2}\right) \bar{\psi}_{R}\left[\left(\partial_{\mu} \partial^{\mu}\right)\left(\gamma_{\nu} A^{\nu}\right)-i\left(m_{R}-m_{N}\right) \partial_{\mu} A^{\mu}\right] \psi_{N}
$$




$$
\begin{aligned}
\mathcal{L}_{\gamma N R}^{(2)} & =-e F_{2}^{R}\left(Q^{2}\right) \bar{\psi}_{R}\left(\sigma_{\mu \nu} \partial^{\mu} A^{\nu}\right) \psi_{N}, \\
\mathcal{L}_{\eta N R} & =-i g_{\eta N R} \bar{\psi}_{R} \gamma_{5} \psi_{N} \phi_{\eta},
\end{aligned}
$$

with $A^{\mu}$ the electromagnetic field, and $\psi_{N}, \psi_{R}, \phi_{\eta}$ the field operators of the nucleon, $P_{11}(1440)$ and $\eta$. With this, the $s$-channel $P_{11}(1440)$ contributions into $B_{i}\left(s, t, Q^{2}\right)$ have the following form:

$$
B_{i}^{R}\left(s, t, Q^{2}\right)=e g_{\eta N R} R_{i}^{R}\left(Q^{2}\right) \frac{1}{s-m_{R}^{2}+i m_{R} \Gamma_{t o t}},
$$

where

$$
\begin{aligned}
& R_{1}^{R}\left(Q^{2}\right)=Q^{2} F_{1}^{R}\left(Q^{2}\right)+\left(m_{N}+m_{R}\right) F_{2}^{R}\left(Q^{2}\right) \\
& R_{2}^{R}\left(Q^{2}\right)=-Q^{2} F_{1}^{R}\left(Q^{2}\right) \\
& R_{3}^{R}\left(Q^{2}\right)=-\frac{Q^{2}}{2} F_{1}^{R}\left(Q^{2}\right) \\
& R_{5}^{R^{\prime}}\left(Q^{2}\right)=\left(m_{R}-m_{N}\right) Q^{2} F_{1}^{R}\left(Q^{2}\right)+\frac{m_{\eta}^{2}-Q^{2}-t}{2} F_{2}^{R}\left(Q^{2}\right) \\
& R_{6}^{R}\left(Q^{2}\right)=2 F_{2}^{R}\left(Q^{2}\right) \\
& R_{8}^{R}\left(Q^{2}\right)=F_{2}^{R}\left(Q^{2}\right) .
\end{aligned}
$$

$\Gamma_{t o t}$ we parametrize according to the formulas presented below, taking the mass and the width of $P_{11}(1440)$ equal to $M=1440 \mathrm{MeV}, \Gamma=350 \mathrm{MeV}$, and the branching ratio into the $\pi N$ channel $\beta_{\pi N}=0.6$. The form factor $F_{2}^{R}\left(Q^{2}\right)$ at $Q^{2}=0$ is related to the $P_{11}(1440) \rightarrow N \gamma$ helicity amplitudes:

$$
A_{1 / 2}=e\left(\pi k \frac{m_{R}}{m_{N}}\right)^{1 / 2} F_{2}^{R}(0),
$$

and can be found from the Particle Data Group [17] values for $A_{1 / 2}$ :

$$
F_{2}^{R}(0) \equiv \frac{\kappa_{N}^{R}}{2 m_{N}}, \kappa_{p}^{R}=-0.5, \kappa_{n}^{R}=0.3 .
$$

The $g_{\eta N N}$ and $g_{\eta N R}$ coupling constants we will found from the analysis of the $\eta$ photoproduction data.

Let us turn now to the imaginary parts of the amplitudes, which we construct through the contributions of the resonances $D_{13}(1520), S_{11}(1535), S_{11}(1650), D_{15}(1675)$, 
$F_{15}(1680), D_{13}(1700), P_{11}(1710), P_{13}(1720)$. The contributions of these resonances into multipole amplitudes we parametrize using the Breit-Wigner formula in the form based on the parametrizations used in Refs. ([13, 16, 18]):

$$
\mathcal{M}\left(W, Q^{2}\right)=\mathcal{M}\left(W=M, Q^{2}\right)\left(\frac{k}{k_{r}}\right)^{n}\left(\frac{q_{\eta, r}}{q_{\eta}} \frac{k_{r}}{k} \frac{\Gamma_{\eta} \Gamma_{\gamma}}{\beta_{\eta N} \Gamma^{2}}\right)^{1 / 2} \frac{M \Gamma}{M^{2}-W^{2}-i M \Gamma_{\text {total }}}
$$

where $n=0$ for $M_{l \pm}, E_{l \pm}, n=1$ for $S_{l \pm}$ and

$$
\begin{aligned}
& \Gamma_{\text {total }}=\Gamma_{\pi}+\Gamma_{\eta}+\Gamma_{\text {inel }}, \\
& \Gamma_{\pi}=\beta_{\pi N} \Gamma\left(\frac{q_{\pi}}{q_{\pi, r}}\right)^{2 l+1}\left(\frac{X^{2}+q_{\pi, r}^{2}}{X^{2}+q_{\pi}^{2}}\right), \\
& \Gamma_{\eta}=\beta_{\eta N} \Gamma\left(\frac{q_{\eta}}{q_{\eta, r}}\right)^{2 l+1}\left(\frac{X^{2}+q_{\eta, r}^{2}}{X^{2}+q_{\eta}^{2}}\right), \\
& \Gamma_{\gamma}=\Gamma\left(\frac{k}{k_{r}}\right)^{2 l^{\prime}+1}\left(\frac{X^{2}+k_{r}^{2}}{X^{2}+k^{2}}\right)^{l^{\prime}}, \\
& \Gamma_{\text {inel }}=\left(1-\beta_{\pi N}-\beta_{\eta N}\right) \Gamma\left(\frac{q_{2 \pi}}{q_{2 \pi, r}}\right)^{2 l+4}\left(\frac{X^{2}+q_{2 \pi, r}^{2}}{X^{2}+q_{2 \pi}^{2}}\right)^{l+2} .
\end{aligned}
$$

For $M_{l \pm}, E_{l+}, S_{l+}, l^{\prime}=l$; for $E_{l-}, S_{l-}, l^{\prime}=l-2$ if $l \geq 2$; for $S_{1-}, l^{\prime}=1 ; M$ and $\Gamma$ are masses and widths of the resonances, $\beta_{\pi N}$ and $\beta_{\eta N}$ are their branching ratios into the $\pi N$ and $\eta N$ channels, $k, q_{\eta} \equiv q, q_{\pi}$ and $q_{2 \pi}$ are the 3 -momenta of the $\gamma, \eta, \pi$ and $2 \pi$ system in the decays of the resonances into the $\gamma N, \eta N, \pi N$ and $2 \pi N$ channels in the c.m.s., $k_{r}, q_{\eta, r}, q_{\pi, r}, q_{2 \pi, r}$ are the magnitudes of these momenta at the resonance peak $(W=M) ; X$ are phenomenological parameters, assumed to be $500 \mathrm{MeV}$ for all resonances, except $S_{11}(1535)$ and $S_{11}(1650)$. For these resonances parameters X were found by fitting experimental data.

Below the thresholds of $2 \pi+N$ and $\eta+N$ productions we take, respectively, $q_{2 \pi}=0$ and $q_{\eta}=0$. So, below $\eta N$ production threshold, $\Gamma_{\text {total }}=\Gamma_{\pi}+\Gamma_{\text {inel }}$, and, respectively, below $2 \pi N$ production threshold, $\Gamma_{\text {total }}=\Gamma_{\pi}$.

Using the parametrizations (10-15) for the contributions of the resonances $S_{11}(1535)$, $S_{11}(1650)$ to $E_{0+}$ and $S_{0+}$, one can easily continue their contributions to the invariant amplitudes to the unphysical region $\left(s<\left(m_{\eta}+m_{N}\right)^{2}\right)$ of the dispersion integrals via the 
formulas:

$$
\begin{aligned}
& \mathcal{E}_{0+} \equiv \frac{E_{0+}}{2 W\left[\left(E_{1}+m_{N}\right)\left(E_{2}+m_{N}\right)\right]^{1 / 2}}, \mathcal{S}_{0+} \equiv \frac{Q^{2} S_{0+}}{2 W\left[\left(E_{1}-m_{N}\right)\left(E_{2}+m_{N}\right)\right]^{1 / 2}}, \\
& B_{1}\left(s, t, Q^{2}\right)=\mathcal{E}_{0+}, \\
& B_{2}\left(s, t, Q^{2}\right)=2 B_{3}\left(s, t, Q^{2}\right)=\frac{-Q^{2}\left(E_{1}+m_{N}\right) \mathcal{E}_{0+}+\left(W-m_{N}\right) \mathcal{S}_{0+}}{2 W k^{2}} \\
& B_{5}\left(s, t, Q^{2}\right)=-\left(W+m_{N}\right) \mathcal{E}_{0+}, \\
& B_{6}\left(s, t, Q^{2}\right)=2 B_{8}\left(s, t, Q^{2}\right)=-\frac{\left(W-m_{N}\right)\left(E_{1}+m_{N}\right) \mathcal{E}_{0+}+\mathcal{S}_{0+}}{W k^{2}} .
\end{aligned}
$$

It is seen that there are no irregularities in the continuation of Eqs. (16) from $s \geq$ $\left(m_{\eta}+m_{N}\right)^{2}$ to $s<\left(m_{\eta}+m_{N}\right)^{2}$.

\section{Isobar model}

The isobar model we use is very close to the model of Ref. [13]. It contains contributions of resonances, and the nonresonance background build from the Born term and the $t$ channel $\rho$ and $\omega$ contributions. Unlike the model of Ref. [13], we take into account not only the resonances with the masses above $\eta N$ production threshold, but also the contribution of the Roper resonance, which, in fact, can be considered as the background contribution. This resonance is introduced in a form described in the previous Section. There is also a difference between our parametrizations of the resonance contributions which consists in the extra factor $W_{R} / W$ in the expressions for $\Gamma_{\pi}$ and $\Gamma_{\eta}$ of Ref. [13], and in the factor $\Gamma_{\gamma} / \Gamma$ in the parentheses of Eq. (10).

We have found the $t$-channel $\rho$ and $\omega$ exchange contributions to $\gamma^{*}+N \rightarrow \eta+N$ by evaluating the Feynman diagrams for this process and the corresponding invariant amplitudes using the effective Lagrangians:

$$
\begin{aligned}
& \mathcal{L}_{\gamma \eta V}=\frac{e \lambda_{V}}{m_{\eta}} F^{(V)}\left(Q^{2}\right) \varepsilon_{\mu \nu \sigma \rho} \partial^{\mu} A^{\nu} \partial^{\sigma} V^{\rho} \phi_{\eta} \\
& \mathcal{L}_{V N N}=\bar{\psi}_{N}\left(g_{1 V} \gamma_{\mu}+\frac{g_{2 V}}{2 m_{N}} \sigma_{\mu \nu} \partial^{\nu}\right) V^{\mu} \psi_{N} .
\end{aligned}
$$


The electromagnetic coupling constants $\lambda_{V}$ are related to the $V \rightarrow \eta \gamma$ radiative decay widths via

$$
\Gamma_{V \rightarrow \eta \gamma}=\frac{e^{2} q_{\eta}^{3}}{12 \pi m_{\eta}^{2}} \lambda_{V}^{2},
$$

where $q_{\eta}$ is the $\eta$ momentum in this decay. The values of $\lambda_{V}$ found using PDG data [17] are presented in Table 1 . The form factors $F^{(V)}\left(Q^{2}\right)$ are introduced to describe the $Q^{2}$ dependence of the $\gamma \eta V$ couplings, at $Q^{2}=0$ they are normalized to $F^{(V)}(0)=1$. The off-shell behaviour of the hadronic couplings were described according to Ref. [13] in the form

$$
g_{i V}=\tilde{g}_{i V}\left(\frac{\Lambda_{V}^{2}-m_{V}^{2}}{\Lambda_{V}^{2}-t}\right)^{2}, i=1,2,
$$

with $\Lambda_{V}=1.3 \mathrm{GeV}$. The contributions of $\rho$ and $\omega$ exchanges to the invariant amplitudes are:

$$
\begin{aligned}
B_{1, V} & =\frac{e \lambda_{V}}{m_{\eta}}\left[2 m_{N} g_{1 V}+t \frac{g_{2 V}}{2 m_{N}}\right] \frac{1}{t-m_{V}^{2}}, \\
B_{2, V} & =\frac{e \lambda_{V}}{m_{\eta}} \frac{g_{2 V}}{4 m_{N}} \frac{Q^{2}+m_{\eta}^{2}-t}{t-m_{V}^{2}}, \\
B_{3, V} & =\frac{e \lambda_{V}}{m_{\eta}} \frac{g_{2 V}}{8 m_{N}} \frac{u-s}{t-m_{V}^{2}}, \quad B_{6, V}=2 \frac{e \lambda_{V}}{m_{\eta}} \frac{g_{1 V}}{t-m_{V}^{2}} .
\end{aligned}
$$

The coupling constants $\tilde{g}_{i V}$ are not well known, their ranges from Ref. [19] are presented in Table 1. In our analysis, we consider them as free parameters. The values of $\tilde{g}_{i V}$ found from the fitting of data on the $\eta$ photoproduction on the protons within the model presented in this Section are given in Table 1.

\section{Results and discussion}

Using the two approaches described in Sections 2 and 3, we have fitted the experimental data on the $\eta$ photoproduction on protons. We have used the information on the differential cross sections from TAPS [11] $\left(W=1.491-1.537 \mathrm{GeV}, E_{\gamma}=716-790 \mathrm{MeV}\right)$, GRAAL [8] $\left(W=1.49-1.716 \mathrm{GeV}, E_{\gamma}=714-1100 \mathrm{MeV}\right)$, and CLAS [9] $(W=$ $1.528-2.12 \mathrm{GeV}, E_{\gamma}=775-1925 \mathrm{MeV}$ ), on the polarized beam asymmetry from 
GRAAL [10] $\left(W=1.506-1.688 \mathrm{GeV}, E_{\gamma}=740-1050 \mathrm{MeV}\right)$, and on the polarized target asymmetry from ELSA [12] $\left(W=1.492-1.719 \mathrm{GeV}, E_{\gamma}=717-1105 \mathrm{MeV}\right)$. As the role of the data, obtained with the polarized beam and target, appeared to be very important, we have restricted our investigation by the energies $W=1.49-1.73 \mathrm{GeV}$, where such data are present.

The fitted parameters in the dispersion relations approach were the coupling constants $g_{\eta N N}, g_{\eta N R}$, and the magnitudes of the multipole amplitudes corresponding to the resonance contributions at the resonance positions. In the isobar model approach there are four additional fitted parameters connected with the the $\rho$ and $\omega$ contributions: $\tilde{g}_{1 \rho}$, $\tilde{g}_{2 \rho}, \tilde{g}_{1 \omega}$ and $\tilde{g}_{2 \omega}$. We have fitted also the masses of all resonances taking into account the ranges given by the PDG [17]. The widths and parameters $\mathrm{X}$ of the most prominent resonances: $S_{11}(1535)$ and $S_{11}(1650)$, were fitted too. The widths of other resonances used in our analysis are presented in Table 2. The used branching ratios correspond to the mean values of the PDG data presented in this Table.

In Table 3 we present the obtained values of the multipole amplitudes for the resonance contributions at the resonance positions. For comparison, multipole amplitudes extracted from the results of Ref. [13] are given. We present also the photoexcitation helicity amplitudes obtained using our results, and the ranges for these amplitudes from the PDG data [17].

In Figs. 1-4 we present our results obtained using both approaches in comparison with the experimental data. The overall $\chi^{2} /$ datapoint was 1.24 and 1.22 in the dispersion relations approach and in the isobar model, respectively.

In Fig. 5 multipole amplitudes are presented.

From Figs. 1-4 it is seen that our results are in very good agreement with the experimental data. The only case, when the experimental data are described not so well, is connected with the target asymmetry at small energies: $E_{\gamma}=717,738 \mathrm{MeV}$, where the data change sign near $90^{\circ}$. Such structure is not seen in both our approaches, it is not described also in other models $[7,13,14]$.

Resonance contributions to the multipole amplitudes. The experimental data on 
the differential cross sections obviously indicate the dominant role of the $s$-wave in the $S_{11}(1535)$ resonance region. These data can be described by the single resonance fit taking into account only the contribution of this resonance. With increasing energy, for good description of the data it is necessary to introduce quite large contribution of the $S_{11}(1650)$ resonance, although its presence visually is seen neither in the experimental data, nor in the multipole amplitude $E_{0+}$ obtained in our analyses (Fig. 5). We have fitted the masses and widths of the $S_{11}(1535)$ and $S_{11}(1650)$, the obtained values are presented in Table 2. It is seen that the Breit-Wigner width of the $S_{11}(1535)$ is modeldependent. The obtained widths of the $S_{11}(1650)$ are smaller than the values given by the PDG. Let us note that the same result was obtained in Ref. [13]. From our results obtained using two approaches and from comparison with the results of Ref. [13] it is seen that the contribution of the resonance $S_{11}(1535)$ to the multipole amplitude $E_{0+}$ is determined very well. It practically does not depend on the used approach and on the difference in the parametrizations of the resonance contributions used in this paper and in Ref. [13]. The results for the $S_{11}(1650)$ contribution to $E_{0+}$ are also quite stable. The sign and order of magnitude of this contribution are determined, however, its absolute value is somewhat different in different approaches.

In the $S_{11}(1535)$ resonance region, where we have $s$-wave dominance, the polarized beam asymmetry is determined by the interference of the $E_{0+}$ and $E_{2-}+M_{2-}$ multipoles only [13]:

$$
\Sigma=3 \sin ^{2} \theta \operatorname{Re}\left[E_{0+}^{*}\left(E_{2-}+M_{2-}\right)\right] /\left|E_{0+}\right|^{2} .
$$

By this reason, having the beam asymmetry data, we are able to find with good accuracy the $D_{13}(1520)$ resonance contribution, although by itself it is very small. Indeed, as it is seen from Table 3, the results for $D_{13}(1520)$ obtained using different approaches are in good agreement with each other.

With increasing energy the beam asymmetry data acquire a forward-backward asymmetry behaviour, which is very sensitive to the $F_{15}(1680)$ contribution $[13,20]$. In order to demonstrate this, we have presented in Fig. 2 by the dotted curve the results for $\Sigma$ at $E_{\gamma}=1050 \mathrm{MeV}$ (in the center of the $F_{15}(1680)$ ) obtained in the isobar model with the 
zero $F_{15}(1680)$ contribution. The sensitivity of $\Sigma$ to the $F_{15}(1680)$ contribution makes it clear, why the values of the $F_{15}(1680)$ multipoles obtained in both approaches are in good agreement with each other. We found that the difference with the results of Ref. [13] is caused by our inclusion into the fitting procedure of the target asymmetry data, which do not present in the analysis of Ref. [13].

The contributions of other resonances $D_{15}(1675), D_{13}(1700), P_{11}(1710), P_{13}(1720)$ are not related immediately to some specific features in the behaviour of the observables $\sigma$, $\Sigma$ and $T$. The results obtained in our analyses using the two approaches, and the comparison with the results of Ref. [13] allow us to make conclusion about the reliability of the obtained $D_{15}(1675), D_{13}(1700), P_{11}(1710), P_{13}(1720)$ contributions to the multipole amplitudes. We have performed also the fitting of the experimental data taking exactly the model of Ref. [13]. It appeared, that the inclusion of the Roper contribution practically does not affect the obtained results, it leads only to a better description of the data with better $\chi^{2}$. However, the inclusion into the analysis of the $\mathrm{T}$ data appeared to be significant. As a result, the conclusion which we make is following. The results for the $D_{15}(1675), P_{11}(1710)$, and $P_{13}(1720)$ do not depend strongly on the used approach. The signs and orders of magnitude of the contributions of these resonances are determined. The strong difference with [13] is connected with the inclusion of the $\mathrm{T}$ data into our analysis. The results for the $D_{13}(1700)$ strongly depend on the model. Even the masses of this resonance, obtained in two approaches, strongly differ from each other (Table 2).

Photoexcitation helicity amplitudes. These amplitudes were calculated using the multipole amplitudes presented in Table 3 by the formulas:

$$
\begin{aligned}
& \tilde{\mathcal{M}}_{l \pm} \equiv \mathcal{M}_{l \pm} \zeta_{\eta N}\left[(2 J+1) \pi \frac{q_{\eta, r}}{k_{r}} \frac{M}{m_{N}} \frac{\Gamma_{t o t}^{2}}{\Gamma_{\eta N}}\right]^{1 / 2} \\
& A_{1 / 2}^{l+}=\frac{1}{2}\left[(l+2) \tilde{E}_{l+}+l \tilde{M}_{l+}\right] \\
& A_{3 / 2}^{l+}=\frac{[l(l+2)]^{1 / 2}}{2}\left(\tilde{M}_{l+}-\tilde{E}_{l+}\right) \\
& A_{1 / 2}^{(l+1)-}=\frac{1}{2}\left[l \tilde{E}_{(l+1)-}-(l+2) \tilde{M}_{(l+1)-}\right]
\end{aligned}
$$




$$
A_{3 / 2}^{(l+1)-}=\frac{[l(l+2)]^{1 / 2}}{2}\left(\tilde{E}_{(l+1)-}+\tilde{M}_{(l+1)-}\right)
$$

where $J$ is the spin of the resonance, and $\zeta_{\eta N}$ is the relative sign between the $N N \eta$ coupling constant in the Born term and the $N^{*} N \eta$ coupling constant, which enter the relation (23) through $\zeta_{\eta N} \Gamma_{\eta N}^{1 / 2} \sim g_{N^{*} N \eta}$. The signs which we have used are presented in Table 3. For the calculation of the photoexcitation helicity amplitudes we have used the masses, widths, and branching ratios given in Table 2. All used branching ratios are within the ranges given by the PDG, except the $P_{13}(1720)$. Here we had to take larger $\beta_{\eta N}$ in order to stay within the ranges for ${ }_{p} A_{1 / 2}$ given by the PDG.

In the case of the $S_{11}(1535)$, there is the difference between the values of the photoexcitation helicity amplitude ${ }_{p} A_{1 / 2}$ obtained using dispersion relations and the isobar model. It is connected with the different values of masses and widths obtained in these approaches. Both values are in good agreement with the results obtained in other analyses of the $\eta$ photoproduction: $95 \div 140 \mu k b^{1 / 2}$ [17]. As in all these analyses our ${ }_{p} A_{1 / 2}$ amplitudes for the $S_{11}(1535) \rightarrow p \gamma$ transition are larger than the values extracted from $\pi$ photoproduction data. However, the extraction of the amplitude ${ }_{p} A_{1 / 2}$ from the multipole amplitudes $E_{0+}$ is connected with the uncertainties caused by the mass, width, and branching ratios of the $S_{11}(1535)$. By this reason, for the comparison of the results obtained in the $\pi$ and $\eta$ photoproduction, it is more reasonable to compare directly the results for the $S_{11}(1535)$ contributions to $E_{0+}$. They are connected by the relation:

$$
E_{0+}^{r e s}(\gamma p \rightarrow \eta p)=E_{0+}^{r e s}(\gamma p \rightarrow \pi p)\left(\frac{q_{\pi, r}}{q_{\eta, r}}\right)^{1 / 2}\left(\frac{\beta_{\eta N}}{\beta_{\pi N}}\right)^{1 / 2}
$$

which does not contain the uncertainty caused by the width of the $S_{11}(1535)$. From the PDG data presented in Table 2 we have:

$$
\left(\frac{q_{\pi, r}}{q_{\eta, r}}\right)^{1 / 2}=1.47 \div 1.71,\left(\frac{\beta_{\eta N}}{\beta_{\pi N}}\right)^{1 / 2}=0.8 \div 1.35 .
$$

So, the maximum value of $E_{0+}^{\text {res }}(\gamma p \rightarrow \eta p)$ which we can obtain from Eq. (25) is

$$
E_{0+}^{r e s, \max }(\gamma p \rightarrow \eta p)=2.3 E_{0+}^{r e s}(\gamma p \rightarrow \pi p) .
$$


The magnitude of $E_{0+}^{r e s}(\gamma p \rightarrow \pi p)$, extracted from the results of Ref. [5] using the BreitWigner formula for the $S_{11}(1535)$ and $S_{11}(1650)$ contributions, is by our estimations $<0.6 \mu k b^{1 / 2}$. This gives $E_{0+}^{\text {res,max }}(\gamma p \rightarrow \eta p)<1.4 \mu k b^{1 / 2}$, which is much smaller than the values in Table 3. Therefore, indeed, there is disagreement between the $S_{11}(1535)$ contributions into $E_{0+}$ extracted from the $\pi$ and $\eta$ photoproduction. It is possible, that this disagreement is connected with the fact that we compare the values which correspond to the so-called "dressed" verteces $\gamma p S_{11}(1535)$, i.e. they contain the contributions caused by the rescattering effects in the background terms. The extraction of the "bare" verteces is model-dependent procedure, which is beyond the scope of this work.

For the resonances $D_{13}(1520)$ and $F_{15}(1680)$, the large photoexcitation helicity amplitudes ${ }_{p} A_{3 / 2}$ are given by the PDG with great accuracy. By this reason having the contributions of these resonances to the $\gamma p \rightarrow \eta p$ multipole amplitudes, we have the possibility to extract the branching ratios for $D_{13}(1520)$ and $F_{15}(1680)$ to the $\eta N$ channel with better accuracy than in the PDG data:

$$
\begin{aligned}
& \Gamma\left(D_{13}(1520) \rightarrow \eta N\right) / \Gamma\left(D_{13}(1520) \rightarrow a l l\right)=0.05 \pm 0.02 \% \\
& \Gamma\left(F_{15}(1680) \rightarrow \eta N\right) / \Gamma\left(F_{15}(1680) \rightarrow a l l\right)=0.16 \pm 0.04 \%
\end{aligned}
$$

The $g_{\eta N N}$ and $g_{\eta N R}$ coupling constants. The values of $g_{\eta N N}$ and $g_{\eta N R}$ found in our analyses are: $g_{\eta N N} /(4 \pi)^{1 / 2}=0.26, g_{\eta N R} /(4 \pi)^{1 / 2}=-1.12$ in the dispersion relations approach, and $g_{\eta N N} /(4 \pi)^{1 / 2}=0.11, g_{\eta N R} /(4 \pi)^{1 / 2}=-0.9$ in the isobar model. These constants are much smaller than $g_{\pi N N}$ and $g_{\pi N R}: g_{\pi N N} /(4 \pi)^{1 / 2}=3.78, g_{\pi N R} /(4 \pi)^{1 / 2}=$ $-3.1 \pm 0.4$. The last value we have found from the width of the $P_{11}(1440) \rightarrow \pi N$ decay, the sign is found from the pion photoproduction data. The small value of $g_{\eta N N}$ was obtained also in the analyses of Refs. [7, 13, 14]. It has explanation in the chiral Lagrangian approach $[21,22]$. The smallness of the $g_{\eta N N}$ and $g_{\eta N R}$ coupling constants leads to the smallness of the $N$ and $P_{11}(1440)$ contributions in comparison with the dominant $S_{11}(1535)$ contribution. However, these contributions, as well the $t$-channel $\rho$ and $\omega$ contributions, which are also small compared to the $S_{11}(1535)$, play an important role in getting the better description of the data with better $\chi^{2}$. In Fig. 5 by the thin dotted 
curves we present the summary background contributions to the multipole amplitudes, caused by the Born term, the $P_{11}(1440)$ resonance and the $\rho$ and $\omega t$-channel exchanges. These results correspond to the isobar model. It is seen that they are very small for all multipole amplitudes, except $M_{1+}$, where the large background is generated mostly by the Born term. As it was mentioned in the Introduction, dispersion relations allow to find the $u$-channel resonance contributions. These contributions are presented for the lowest multipoles in Fig. 5 by the thin dashed curves. For the multipoles with $l>1$ they are negligibly small. It is seen that the $u$-channel resonance contributions are very small for all multipoles, except $E_{0+}$.

Recently the properties of the $P_{11}(1710)$ resonance have been discussed in connection with its possible identification as the member of the baryon anti-decuplet in the chiral solitons picture $[23,24,25]$. If this identification is right, the width of the $P_{11}(1710)$ should be $\simeq 40 \mathrm{MeV}[23]$, and the magnetic moment of the transition $P_{11}(1710) \rightarrow \gamma N$, $\mu_{N N^{*}} \equiv \frac{\kappa_{N N^{*}}}{2 m_{N}}$, should be in the ranges [24]:

$$
\kappa_{p p^{*}}=-0.15 \div 0.15, \kappa_{n n^{*}}=-1 \div-0.3
$$

We performed the fit of the experimental data on the proton, taking $\Gamma\left(P_{11}(1710)\right)=$ $40 \mathrm{MeV}$. It appeared, that the results were changed only slightly. The magnetic moment of the $P_{11}^{+}(1710) \rightarrow \gamma p$ transition found using Eqs. $(8,9)$ is equal to

$$
\kappa_{p p^{*}}=0.05 \div 0.06
$$

which is within the ranges (30). The preliminary data on the $\eta$ photoproduction on the neutron obtained at large angles $120^{\circ}, 140^{\circ}$, and $160^{\circ}$ show a sharp rise of the ratio $\sigma(\gamma n \rightarrow \eta n) / \sigma(\gamma p \rightarrow \eta p)$ in the vicinity of the $P_{11}(1710)[25]$. According to our very approximate estimations, such behaviour of the cross sections can be described taking

$$
\kappa_{n n^{*}}=-0.3
$$

This means that $\left|\kappa_{p p^{*}}\right| \ll\left|\kappa_{n n^{*}}\right|$, as it is predicted by the chiral solitons picture. So, more complete data on the $\eta$ photoproduction on the neutron, which will allow to make more 
reliable analysis, may provide very interesting possibility to find arguments in favour of the identification of the $P_{11}(1710)$ resonance as the member of the baryon anti-decuplet.

In the analysis of Ref. [14] of the $\gamma p \rightarrow \eta p$ data the need for a third $S_{11}$ resonance in the second resonance region was found. Such a resonance has been discussed in Ref. [26]. However, in both our approaches such state is not supported by the fits to the data.

\section{Conclusion}

In this paper we presented the results of the analysis of $\eta$ photoproduction on protons, performed using data on cross sections from TAPS [11], GRAAL [8] and CLAS [9], on beam asymmetry from GRAAL [10], and on target asymmetry from ELSA [12]. The analysis is made using both fixed- $t$ dispersion relations and an isobar model. The isobar model we use is very close to that of Ref. [13]. The difference lies in a different parametrization of the resonance contributions and in our inclusion of the $P_{11}(1440)$ contribution in the model. Another difference with the analysis of Ref. [13] is the inclusion of the target asymmetry data in our fitting procedure.

The utilization of two approaches and comparison with the results of Ref. [13] allow us to clarify the model-dependence of the results obtained. We conclude that the results for the contributions of the resonances $D_{13}(1520), S_{11}(1535), F_{15}(1680)$ to the corresponding multipole amplitudes are stable, and do not depend on the model used. This is connected with the fact that the $D_{13}(1520), S_{11}(1535), F_{15}(1680)$ contributions are related directly to specific features of the cross section and beam asymmetry data. Determination of the $D_{13}(1520)$ and $F_{15}(1680)$ resonance contributions with good accuracy, in combination with the PDG results for the ${ }_{p} A_{3 / 2}\left(D_{13}(1520) \rightarrow \gamma N\right)$ and ${ }_{p} A_{3 / 2}\left(F_{15}(1680) \rightarrow \gamma N\right)$ helicity amplitudes, allowed us to find the branching ratios for $D_{13}(1520) \rightarrow \eta N, F_{15}(1680) \rightarrow \eta N$ decays with significantly better accuracy than the PDG data.

The total Breit-Wigner width of the $S_{11}(1535)$ is model-dependent; we have obtained $\Gamma\left(S_{11}(1520)\right)=142$ and $195 \mathrm{MeV}$, using dispersion relations and the isobar model 
respectively. The photoexcitation helicity amplitudes ${ }_{p} A_{1 / 2}$ for the $S_{11}(1535) \rightarrow p \gamma$ transition obtained in both approaches are in good agreement with the results obtained in other analyses of the $\eta$ photoproduction data.

The contributions of other resonances are not related directly to the specific features of the observables used in the fitting procedure. For this reason the results for these resonances are more model-dependent. With this, the signs and orders of magnitude of the $S_{11}(1650), D_{15}(1675), P_{11}(1710), P_{13}(1720)$ contributions to the corresponding multipole amplitudes are determined. The results for the $D_{13}(1700)$ are strongly modeldependent.

The $\eta N$ branching ratios for all resonances, obtained combining the PDG photoexcitation helicity amplitudes and the resonance contributions to multipole amplitudes from our analysis, are within the ranges given by the PDG. The only exception is the $P_{13}(1720)$ resonance. Here we had to take larger $\beta_{\eta N}=18-25 \%$ in order to stay within the ranges for ${ }_{p} A_{1 / 2}$ given by the PDG.

\section{Acknowledgments}

I am thankful to V. D. Burkert, who has initiated my interest to this investigation. I am grateful to L. Tiator for very useful correspondence, to S. Dytman and V. Mokeev for useful discissions, and to M. Dugger and Wen-Tai Chiang for their help in getting data. I express my gratitude for the hospitality at Jefferson Lab where the last stage of this work was accomplished.

\section{Appendix A. Relations between invariant and mul- tipole amplitudes}

In order to connect invariant and multipole amplitudes it is convenient to introduce the intermediate amplitudes $f_{i}\left(s, \cos \theta, Q^{2}\right)$ :

$$
f_{1}=\left[\left(W-m_{N}\right) B_{1}-B_{5}\right] \frac{\left[\left(E_{1}+m_{N}\right)\left(E_{2}+m_{N}\right)\right]^{1 / 2}}{8 \pi W}
$$




$$
\begin{aligned}
f_{2}= & {\left[-\left(W+m_{N}\right) B_{1}-B_{5}\right] \frac{\left[\left(E_{1}-m_{N}\right)\left(E_{2}-m_{N}\right)\right]^{1 / 2}}{8 \pi W}, } \\
f_{3}= & {\left[2 B_{3}-B_{2}+\left(W+m_{N}\right)\left(\frac{B_{6}}{2}-B_{8}\right)\right] \frac{\left[\left(E_{1}-m_{N}\right)\left(E_{2}-m_{N}\right)\right]^{1 / 2}\left(E_{2}+m\right)}{8 \pi W}, } \\
f_{4}= & {\left[-\left(2 B_{3}-B_{2}\right)+\left(W-m_{N}\right)\left(\frac{B_{6}}{2}-B_{8}\right)\right] \frac{\left[\left(E_{1}+m_{N}\right)\left(E_{2}+m_{N}\right)\right]^{1 / 2}\left(E_{2}-m\right)}{8 \pi W}, } \\
f_{5}=\left\{\left[Q^{2} B_{1}+\left(W-m_{N}\right) B_{5}+2 W\left(E_{1}-m_{N}\right)\left(B_{2}-\frac{W+m_{N}}{2} B_{6}\right)\right]\left(E_{1}+m_{N}\right)\right. & \left.-X\left[\left(2 B_{3}-B_{2}\right)+\left(W+m_{N}\right)\left(\frac{B_{6}}{2}-B_{8}\right)\right]\right\} \frac{\left(E_{1}-m_{N}\right)\left(E_{2}+m_{N}\right)}{8 \pi W Q^{2}}, \\
& \left\{-\left[Q^{2} B_{1}-\left(W+m_{N}\right) B_{5}+2 W\left(E_{1}+m_{N}\right)\left(B_{2}+\frac{W-m_{N}}{2} B_{6}\right)\right]\left(E_{1}-m_{N}\right)\right. \\
f_{6}=\quad & \left.+X\left[\left(2 B_{3}-B_{2}\right)-\left(W-m_{N}\right)\left(\frac{B_{6}}{2}-B_{8}\right)\right]\right\} \frac{\left(E_{1}+m_{N}\right)\left(E_{2}-m_{N}\right)}{8 \pi W Q^{2}},
\end{aligned}
$$

where

$$
X=\frac{\tilde{k}_{0}}{2}\left(t-m_{\eta}^{2}+Q^{2}\right)-Q^{2} \tilde{q}_{0}
$$

$\theta$ is the polar angle of $\eta$ in the c.m.s., $\tilde{k}_{0}, \tilde{q}_{0}, E_{1}, E_{2}$ are the energies of the virtual photon, eta, initial and final nucleons in this system.

The expansion of the intermediate amplitudes over multipole amplitudes $M_{l \pm}\left(s, Q^{2}\right)$, $E_{l \pm}\left(s, Q^{2}\right), S_{l \pm}\left(s, Q^{2}\right)$ has the form:

$$
\begin{aligned}
f_{1} & =\sum\left\{\left(l M_{l+}+E_{l+}\right) P_{l+1}^{\prime}(\cos \theta)+\left[(l+1) M_{l-}+E_{l-}\right] P_{l-1}^{\prime}(\cos \theta)\right\} \\
f_{2} & =\sum\left[(l+1) M_{l+}+l M_{l-}\right] P_{l}^{\prime}(\cos \theta) \\
f_{3} & =\sum\left[\left(E_{l+}-M_{l+}\right) P_{l+1}^{\prime \prime}(\cos \theta)+\left(E_{l-}+M_{l-}\right) P_{l-1}^{\prime \prime}(\cos \theta)\right] \\
f_{4} & =\sum\left(M_{l+}-E_{l+}-M_{l-}-E_{l-}\right) P_{l}^{\prime \prime}(\cos \theta) \\
f_{5} & =\sum\left[(l+1) S_{l+} P_{l+1}^{\prime}(\cos \theta)-l S_{l-} P_{l-1}^{\prime}(\cos \theta)\right] \\
f_{6} & =\sum\left[l S_{l-}-(l+1) S_{l+}\right] P_{l}^{\prime}(\cos \theta)
\end{aligned}
$$

The amplitudes $f_{i}\left(s, \cos \theta, Q^{2}\right)$ are related to the helicity amplitudes by:

$$
\begin{aligned}
& H_{1}=-\cos \frac{\theta}{2} \sin \theta\left(f_{3}+f_{4}\right) / \sqrt{2}, \\
& H_{2}=-\sqrt{2} \cos \frac{\theta}{2}\left[f_{1}-f_{2}-\sin ^{2} \frac{\theta}{2}\left(f_{3}-f_{4}\right)\right],
\end{aligned}
$$




$$
\begin{aligned}
& H_{3}=\sin \frac{\theta}{2} \sin \theta\left(f_{3}-f_{4}\right) / \sqrt{2}, \\
& H_{4}=\sqrt{2} \sin \frac{\theta}{2}\left[f_{1}+f_{2}+\cos ^{2} \frac{\theta}{2}\left(f_{3}+f_{4}\right)\right], \\
& H_{5}=-\frac{Q}{k} \cos \frac{\theta}{2}\left(f_{5}+f_{6}\right), \\
& H_{6}=\frac{Q}{k} \sin \frac{\theta}{2}\left(f_{5}-f_{6}\right) .
\end{aligned}
$$

In the case of the photoproduction, the differential cross section, the polarization of the final nucleon, and the polarized beam and target asymmetries are related to the helicity amplitudes in the following way:

$$
\begin{aligned}
\sigma & \equiv \frac{d \sigma}{d \Omega}=\frac{1}{2} \frac{q}{k}\left(\left|H_{1}\right|^{2}+\left|H_{2}\right|^{2}+\left|H_{3}\right|^{2}+\left|H_{4}\right|^{2}\right), \\
P & =-\frac{q}{k} \frac{1}{\sigma} \mathcal{I} m\left(H_{1} H_{3}^{*}+H_{2} H_{4}^{*}\right), \\
\Sigma & =\frac{q}{k} \frac{1}{\sigma} \mathcal{R} e\left(H_{1} H_{4}^{*}-H_{2} H_{3}^{*}\right), \\
T & =\frac{q}{k} \frac{1}{\sigma} \mathcal{I} m\left(H_{1} H_{2}^{*}+H_{3} H_{4}^{*}\right) .
\end{aligned}
$$

\section{References}

[1] T.P. Vrana, S.A. Dytman, and T.-S.H.Lee, Phys. Rept. 328, 181 (2000).

[2] T. Feuster and U. Mosel, Phys. Rev. C58, 457 (1998).

[3] D. M. Manley and E. M. Saleski, Phys. Rev. D45, 4002 (1992).

[4] R. A. Arndt, I. I. Strakovski, R. L. Workman, and M. M. Pavan, Phys. Rev. C52, 2120 (1995).

[5] R. A. Arndt, W. J. Briscoe, I. I. Strakovski, and R. L. Workman, Phys. Rev. C66, 055213 (2002). 
[6] D. Drechsel, O. Hanstein, S. S. Kamalov, and L. Tiator, Nucl. Phys. A645, 145 (1999).

[7] T. Feuster and U. Mosel, Phys.Rev. C59, 460 (1999).

[8] F. Renard et al., Phys. Lett. B528, 215 (2002).

[9] M. Dugger et al., Phys. Rev. Lett. 89, 222002 (2002).

[10] J. Ajaka et al., Phys. Rev. Lett. 81, 1797 (1998).

[11] B. Krusche et al., Phys. Rev. Lett. 74, 3736 (1995).

[12] A. Bock et al., Phys. Rev. Lett. 81, 534 (1998).

[13] Wen-Tai Chiang, Shin Nan Yang, L.Tiator, and D.Drechsel, Nucl.Phys. A700, 429 (2002).

[14] B. Saghai and Z. Li, Eur. Phys. J. A11, 217 (2001).

[15] I.G.Aznauryan, Phys. Rev. C67, 015209 (2003).

[16] R. C. E. Devenish and D. H. Lyth, Phys. Rev. D5, 47 (1972).

[17] Review of Particle Physics, Phys. Rev. D66 (2002).

[18] R.L. Walker, Phys. Rev. 182, 1729 (1969).

[19] O.Dumbrajs et al., Nucl.Phys. B216, 277 (1983).

[20] L.Tiator, D. Drechsel, G. Knochlein, and C. Bennhold, Phys. Rev. C60, 035210 (1999).

[21] M. Kirchbach and L.Tiator, Nucl.Phys. A604, 385 (1996).

[22] S. Neumeir and M. Kirchbach, Int. J. Mod.Phys. A15, 4325 (2000).

[23] D. Diakonov, V. Petrov, and M. V. Polyakov, Z. Phys. A359, 305 (1997). 
[24] M. V. Polyakov and A. Rathke, hep-ph/0033138.

[25] V. Kouznetsov, Proceedings of the Workshop NSTAR2002.

[26] Z.Li, R. Workman, Phys. Rev. C53, R549 (1996). 


\section{Figure Captions}

Fig. 1. Differential cross section for $\gamma p \rightarrow \eta p$. The solid and dashed curves are the results obtained using dispersion relations and isobar model. The data are from TAPS [11] (open circles), GRAAL [8] (full squares), and CLAS [9] (full triangles).

Fig. 2. Polarized beam asymmetry for $\gamma p \rightarrow \eta p$. The solid and dashed curves are the results obtained using dispersion relations and isobar model. The dotted curve at $E_{\gamma}=1050 \mathrm{MeV}$ are the results of the isobar model with the $F_{15}(1680)$ contribution taken to be equal 0 . The data are from GRAAL [10].

Fig. 3. Polarized target asymmetry for $\gamma p \rightarrow \eta p$. The solid and dashed curves are the results obtained using dispersion relations and isobar model. The data are from ELSA [12].

Fig. 4. Total cross section for $\gamma p \rightarrow \eta p$. The solid and dotted curves are the results obtained using dispersion relations and isobar model. The data are from TAPS [11] (open circles), GRAAL [8] (full circles), and CLAS [9] (open squares).

Fig. 5. The results for the multipole amplitudes. Solid (dotted) and dashed (dashdotted) curves are the real and imaginary parts of the amplitudes obtained using dispersion relations (isobar model). By the thin dotted curves the isobar model background contributions caused by the Born term, the $P_{11}(1440)$ resonance and the $\rho$ and $\omega t$ channel exchanges are presented. By the thin dashed curves the $u$-channel resonance contributions found using dispersion relations are presented.

\section{Table Captions}

Table 2. Masses, widths, and branching ratios of the resonances. In the first rows the ranges given by the PDG [17] are presented. In the second rows we list the widths used in our analysis. The masses and widths marked by the stars are found from the fitting of the data: on the second rows using dispersion relations and on the third rows using the 
isobar model. The branching ratios $\beta_{\eta N}$ in the second and third rows are used for the calculation of the photoexcitation helicity amplitudes presented in Table 3.

Table 3. Resonance contributions into the multipole amplitudes at the resonance positions (in $\mu b^{1 / 2}$ ) and photoexcitation helicity amplitudes (in $10^{-3} \mathrm{GeV}^{-1 / 2}$ ). The values on the first and second rows are found in this work using dispersion relations and isobar model, respectively. The values on the third rows for the multipole amplitudes are from Ref. [13]; for the photoexcitation helicity amplitudes on the third rows the PDG data [17] are presented. $\zeta_{\eta N}$ are the relative signs between the $N N \eta$ and $N^{*} N \eta$ coupling constants which are used for the calculation of the photoexcitation helicity amplitudes by the relation (23). 


\begin{tabular}{ccccc}
\hline$V$ & $m_{V}(\mathrm{MeV})$ & $\tilde{g}_{1}$ & $\tilde{g}_{2} / \tilde{g}_{1}$ & $\lambda_{V}$ \\
\hline$\rho$ & 768.5 & $1.8-3.2$ & $4.3-6.6$ & $1.02 \pm 0.09$ \\
& & 1.6 & 5.4 & \\
$\omega$ & \multirow{2}{*}{782.6} & $8-14$ & $0-(-1)$ & $0.29 \pm .03$ \\
& & 14.7 & -0.55 & \\
\hline
\end{tabular}

Table 1: Parameters for the vector mesons. The ranges for $\tilde{g}_{1,2}$ are from Ref. [19], the values of $\lambda_{V}$ are extracted from the PDG data [17] for $V \rightarrow \eta \gamma$. The values on the second rows are obtained in this work from the analysis of the $\gamma p \rightarrow \eta p$ data using the isobar model. 


\begin{tabular}{|c|c|c|c|c|}
\hline Resonance & $M(M e V)$ & $\Gamma(\mathrm{MeV})$ & $\beta_{\eta N}(\%)$ & $\beta_{\pi N}(\%)$ \\
\hline \multirow[t]{3}{*}{$D_{13}(1520)$} & $1515-1530$ & $110-135$ & $0 \pm 1$ & $50-60$ \\
\hline & $1510^{*}$ & 120 & 0.032 & \\
\hline & $1523^{*}$ & & 0.073 & \\
\hline \multirow[t]{3}{*}{$S_{11}(1535)$} & $1520-1555$ & $100-200$ & $30-55$ & $35-55$ \\
\hline & $1527^{*}$ & $142^{*}$ & 50 & \\
\hline & $1542^{*}$ & $195^{*}$ & 50 & \\
\hline \multirow[t]{3}{*}{$S_{11}(1650)$} & $1640-1680$ & $145-190$ & $3-10$ & $55-90$ \\
\hline & $1648^{*}$ & $85^{*}$ & 3.8 & \\
\hline & $1649^{*}$ & $125^{*}$ & 5.5 & \\
\hline \multirow[t]{3}{*}{$D_{15}(1675)$} & $1670-1685$ & $140-180$ & $0 \pm 1$ & $40-50$ \\
\hline & $1666^{*}$ & 160 & 1.2 & \\
\hline & $1663^{*}$ & & 1.4 & \\
\hline \multirow[t]{3}{*}{$F_{15}(1680)$} & 1675-1690 & $120-140$ & $0 \pm 1$ & $60-70$ \\
\hline & $1670^{*}$ & 130 & 0.13 & \\
\hline & $1676^{*}$ & & 0.18 & \\
\hline \multirow[t]{3}{*}{$D_{13}(1700)$} & $1650-1750$ & $50-150$ & $0 \pm 1$ & $5-15$ \\
\hline & $1634^{*}$ & 100 & 1 & \\
\hline & $1727^{*}$ & & 1 & \\
\hline \multirow[t]{3}{*}{$P_{11}(1710)$} & $1680-1740$ & $50-250$ & $6 \pm 1$ & $10-20$ \\
\hline & $1710^{*}$ & 100 & 7 & \\
\hline & $1740^{*}$ & & 6 & \\
\hline \multirow[t]{3}{*}{$P_{13}(1720)$} & $1650-1750$ & $100-200$ & $4 \pm 1$ & $10-20$ \\
\hline & $1720^{*}$ & 150 & 25 & \\
\hline & $1750^{*}$ & & 18 & \\
\hline
\end{tabular}

Table 2: 


\begin{tabular}{|c|c|c|c|c|c|}
\hline Resonance & $E_{l \pm}$ & $M_{l \pm}$ & ${ }_{p} A_{1 / 2}$ & ${ }_{p} A_{3 / 2}$ & $\zeta_{\eta N}$ \\
\hline \multirow[t]{3}{*}{$D_{13}(1520)$} & 0.053 & 0.029 & -40 & 166 & +1 \\
\hline & 0.071 & 0.040 & -43 & 166 & +1 \\
\hline & 0.062 & 0.038 & $-24 \pm 9$ & $166 \pm 5$ & \\
\hline \multirow{3}{*}{$S_{11}(1535)$} & 1.853 & & 96 & & +1 \\
\hline & 1.832 & & 119 & & +1 \\
\hline & 1.845 & & $90 \pm 30$ & & \\
\hline \multirow[t]{3}{*}{$S_{11}(1650)$} & -0.268 & & 53 & & -1 \\
\hline & -0.411 & & 53 & & -1 \\
\hline & -0.437 & & $53 \pm 16$ & & \\
\hline \multirow[t]{3}{*}{$D_{15}(1675)$} & -0.012 & -0.008 & 27 & -4 & -1 \\
\hline & -0.011 & -0.012 & 27 & 1 & -1 \\
\hline & 0 & 0.078 & $19 \pm 8$ & $15 \pm 9$ & \\
\hline \multirow[t]{3}{*}{$F_{15}(1680)$} & 0.024 & 0.015 & -15 & 133 & +1 \\
\hline & 0.029 & 0.018 & -14 & 133 & +1 \\
\hline & 0.015 & 0.01 & $-14 \pm 6$ & $133 \pm 12$ & \\
\hline \multirow[t]{3}{*}{$D_{13}(1700)$} & 0.015 & -0.029 & -30 & 7 & -1 \\
\hline & 0.001 & -0.001 & -1 & 0 & -1 \\
\hline & 0.009 & -0.007 & $-18 \pm 13$ & $-2 \pm 24$ & \\
\hline \multirow[t]{3}{*}{$P_{11}(1710)$} & & 0.172 & 29 & & -1 \\
\hline & & 0.127 & 24 & & -1 \\
\hline & & -0.26 & $9 \pm 22$ & & \\
\hline \multirow[t]{3}{*}{$P_{13}(1720)$} & 0.138 & 0.199 & 48 & 8 & +1 \\
\hline & 0.126 & 0.124 & 48 & 0 & +1 \\
\hline & 0.032 & -0.016 & $18 \pm 30$ & $-19 \pm 20$ & \\
\hline
\end{tabular}

Table 3: 

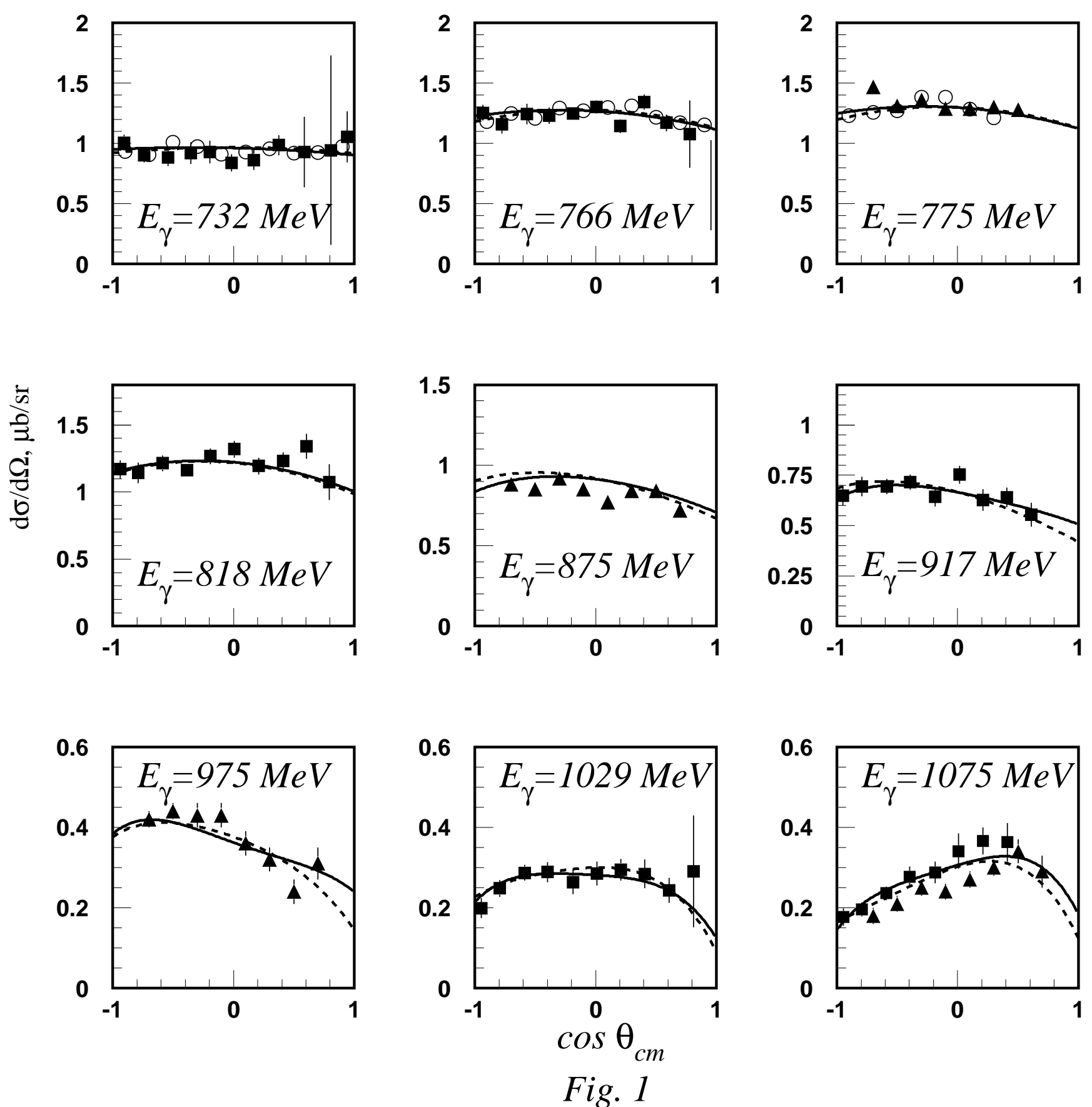

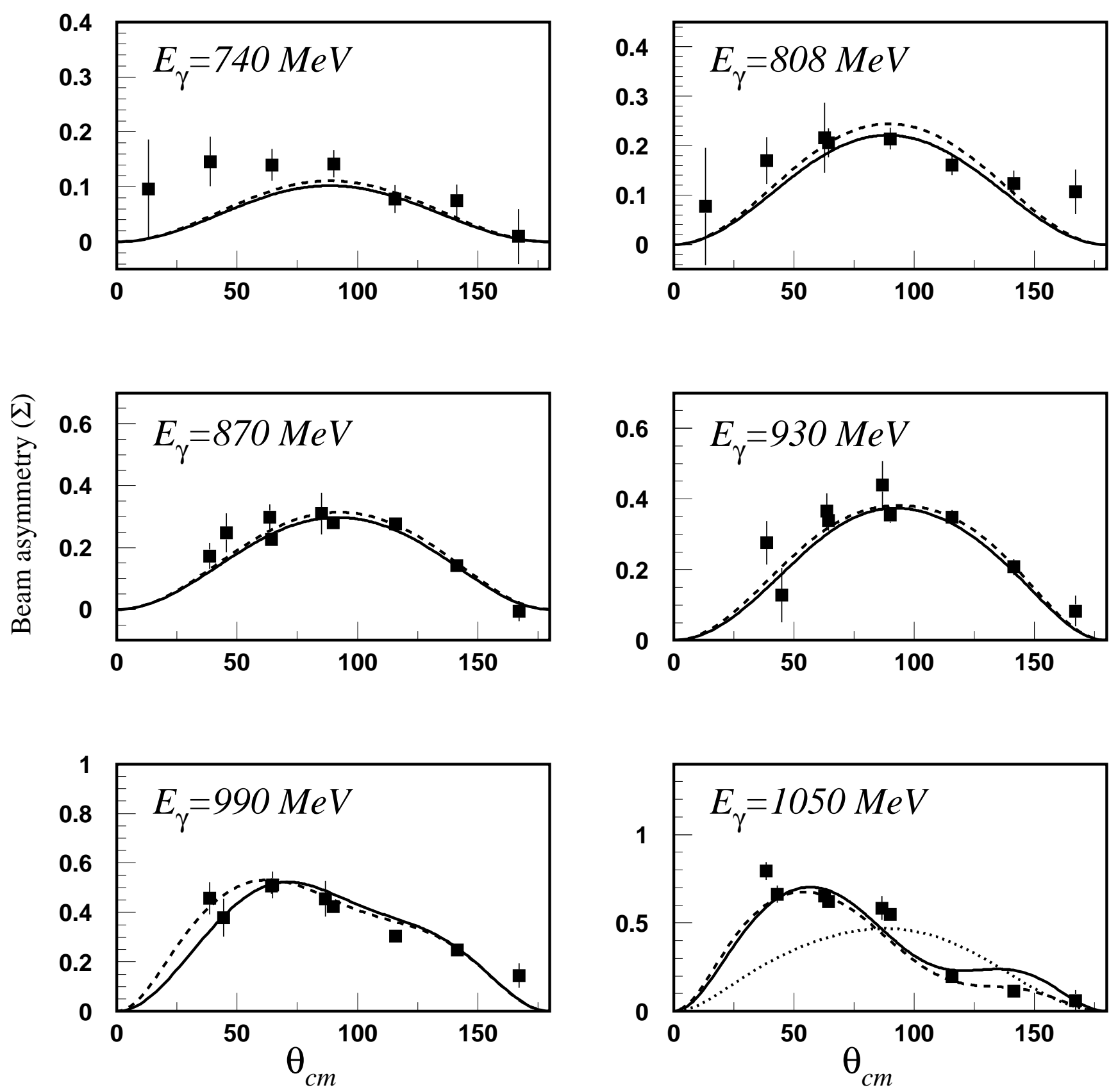

Fig. 2 

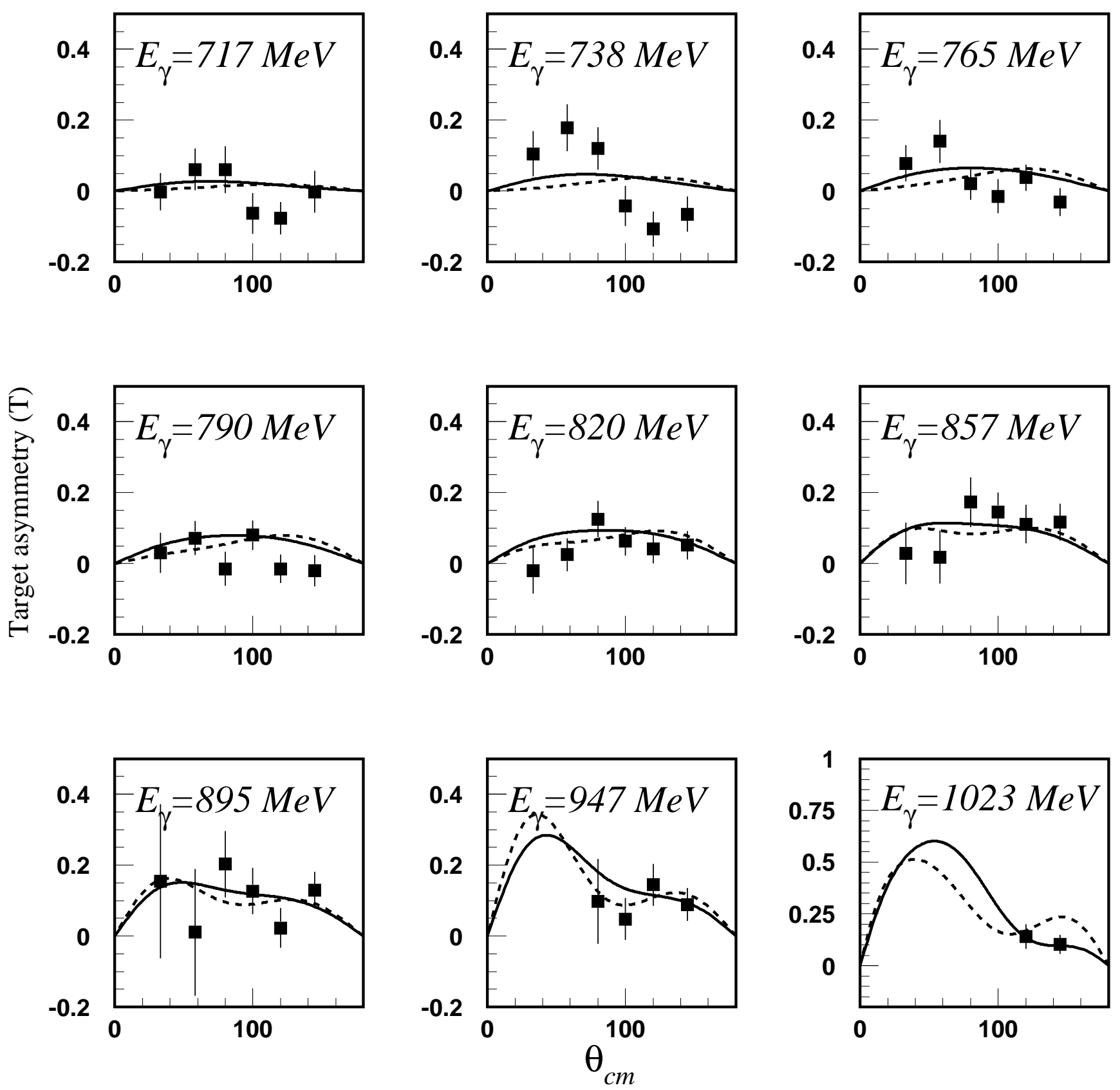

Fig. 3 


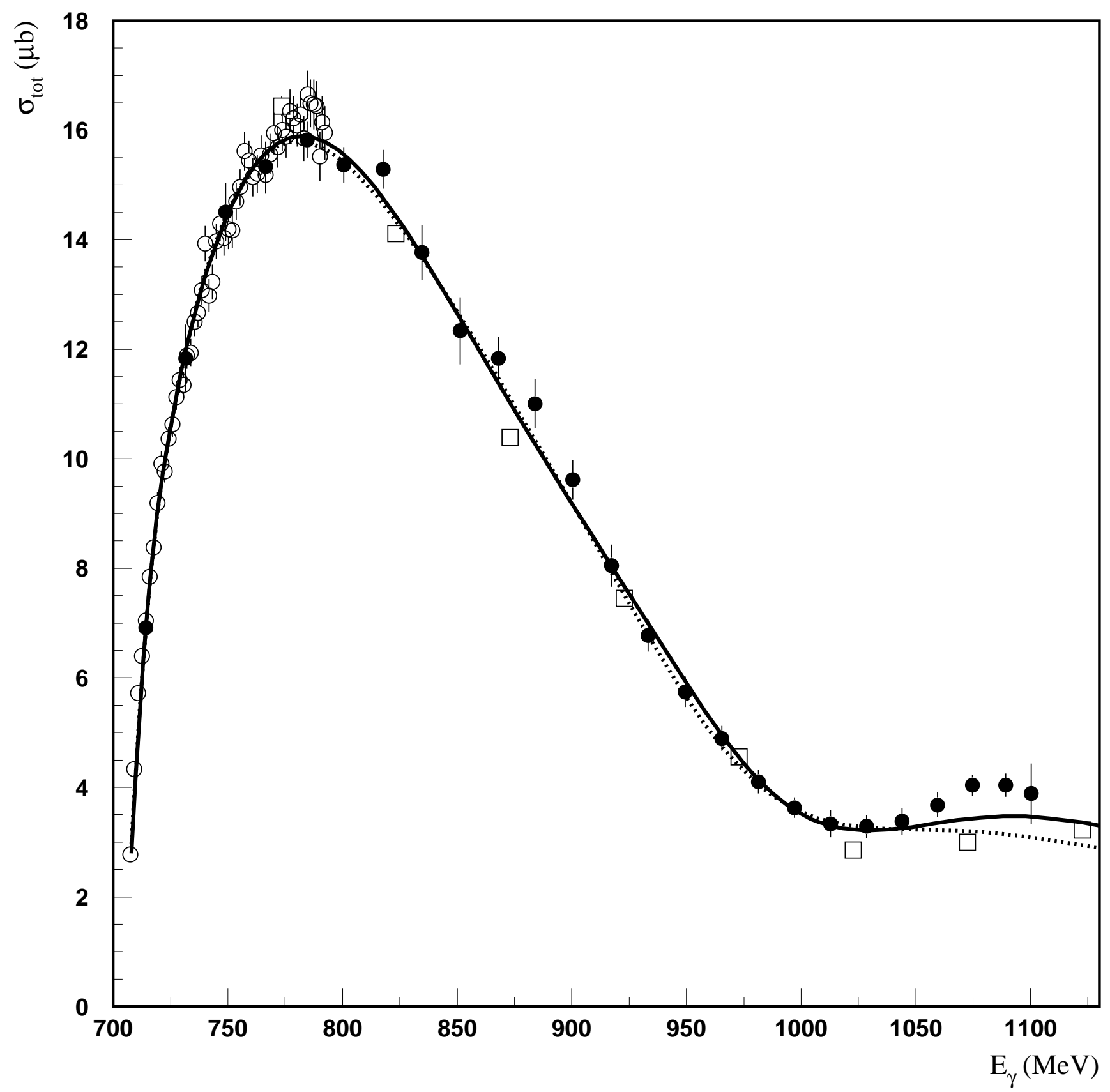

Fig. 4 

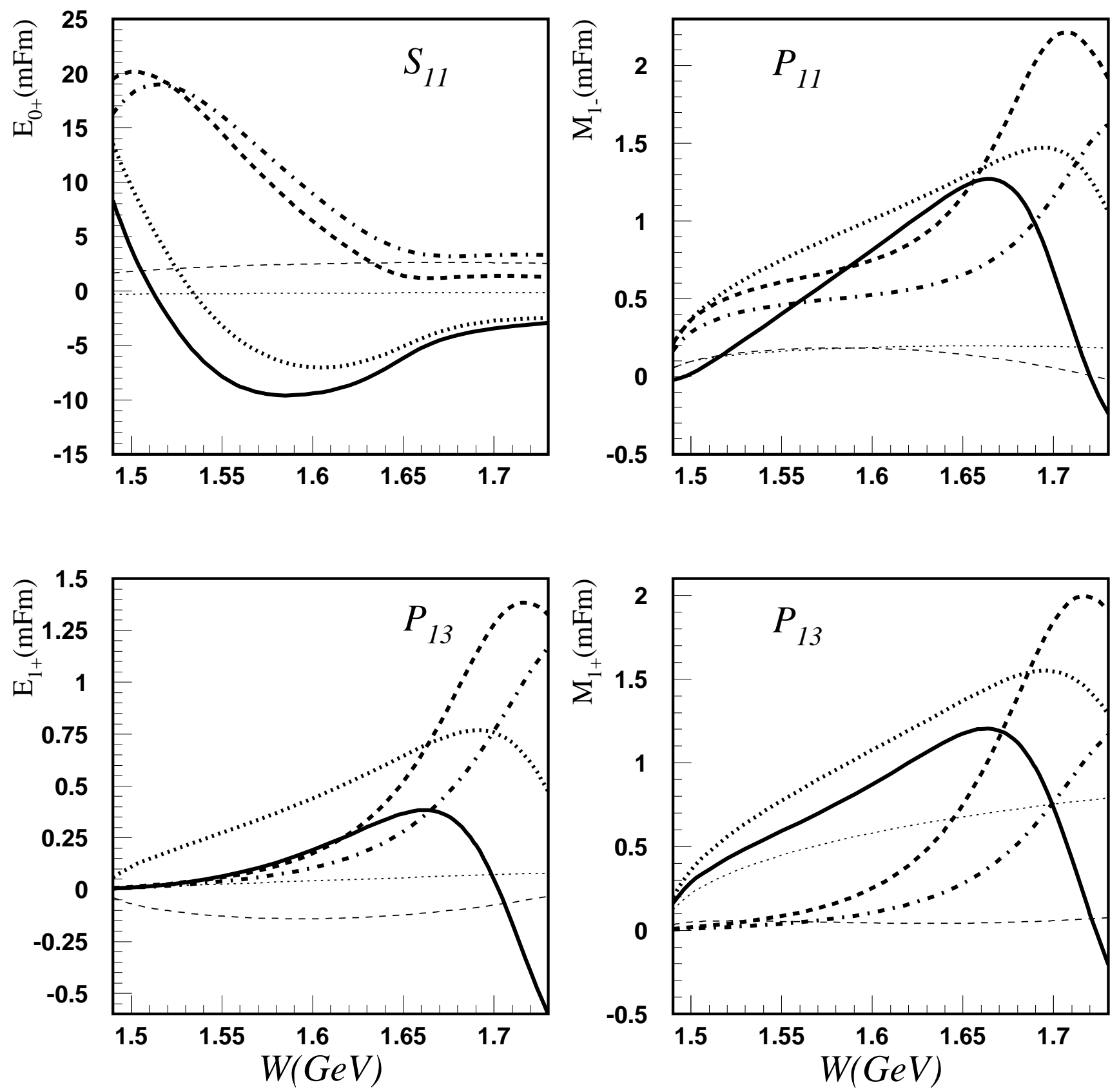

Fig. 5 

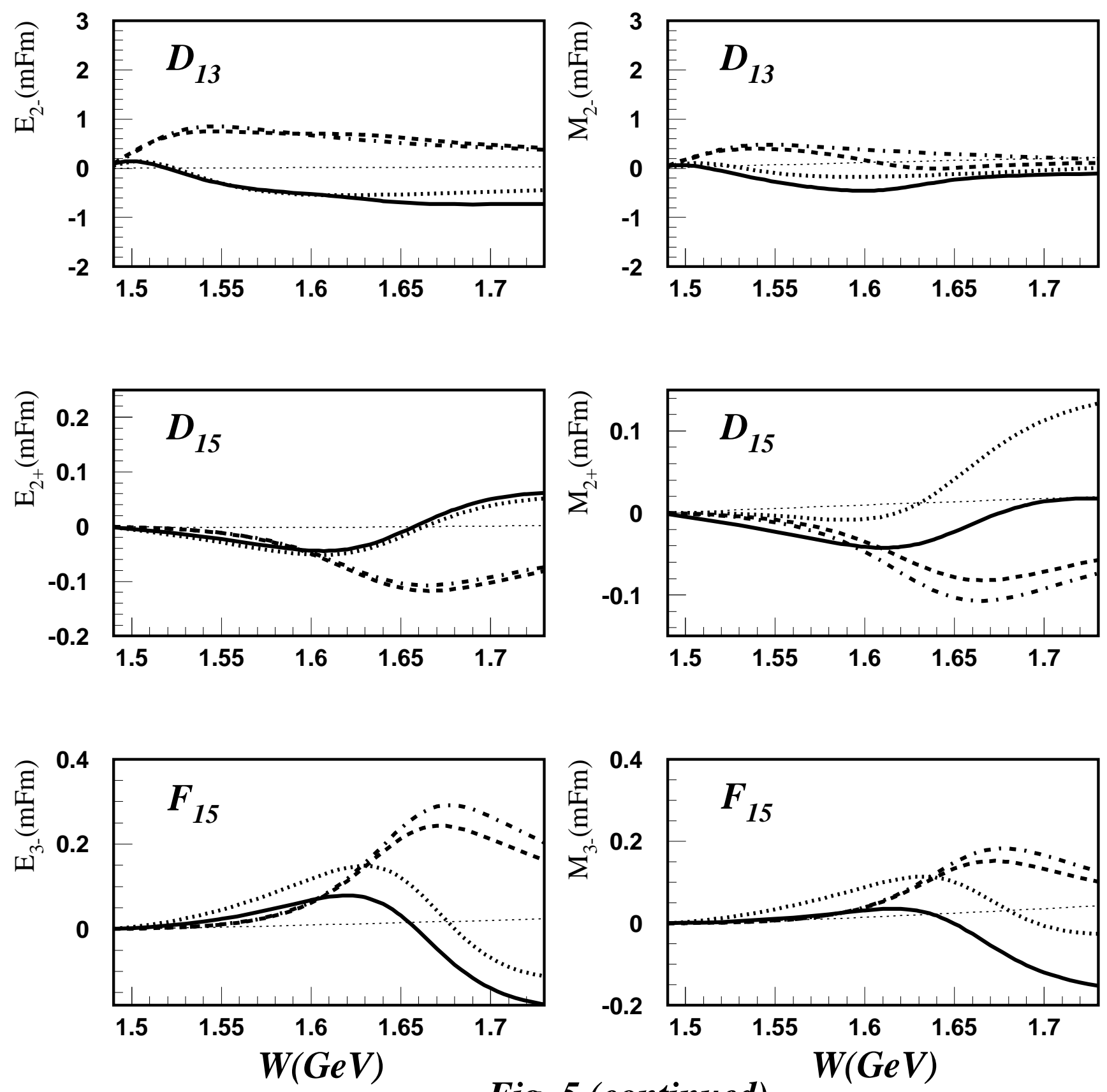

Fig. 5 (continued) 